ARTICLE OPEN

\title{
Ageing properties and polymer/fuel interactions of polyamide 12 exposed to (bio)diesel at high temperature
}

\author{
Xin-Feng Wei ${ }^{1}$, Loris De Vico ${ }^{1}$, Pierre Larroche ${ }^{1}$, Kai J. Kallio ${ }^{2}$, Stefan Bruder ${ }^{3}$, Martin Bellander ${ }^{3}$, Ulf W. Gedde ${ }^{1}$ and \\ Mikael S. Hedenqvist ${ }^{1}$
}

Biodiesel derived from oil crops and animal fats has been developed as a promising carbon-neutral alternative to petroleum fuels in the transport sector, but the compatibility between biodiesel/petroleum diesel and polymer components in the automotive fuel system has not been free from controversy. In this present study, the degradation of polyamide 12 (PA12), one of the most common polymers used in vehicle fuel systems, has been investigated after exposure to petroleum diesel, biodiesel and a mixture of these ( $20 \mathrm{vol} . \%$ of biodiesel/80 vol.\% petroleum diesel). Fuel sorption kinetics, glass transition temperature data and mechanical properties all showed that the fuels plasticized the PA12. In addition, monomers and oligomers were extracted from PA12 by the fuels. The long-term exposure led to oxidation and an annealing-induced increase in crystallinity of the polymer. The plasticization, oxidation and annealing effects were combined with the tensile mechanical properties to assess the overall degree of ageing and degradation of the PA12 material. The fuel-polymer interactions and ageing mechanisms, demonstrated here at high temperature for PA12, are 'generic' in the sense that they are also expected to occur, to various degrees, with many other polymers and they indicate that care should be taken when choosing polymers in applications where they will be exposed to fuels at high temperature.

npj Materials Degradation (2019)3:1 ; https://doi.org/10.1038/s41529-018-0065-y

\section{INTRODUCTION}

Polymer components in motor vehicle fuel-delivery systems undergo thermal stress and fuel attack, which may cause severe deterioration in performance and operational problems such as fuel leakage, engine knocking and fuel pump failure. ${ }^{1,2}$ In recent years, the degradation of polymers in vehicle fuel systems has gained increasing attention due to the increasing use of biodiesel in the transport sector. ${ }^{3-6}$ Many countries have mandated the use of biodiesel in blends with petroleum diesel, in some regions up to 20 vol.\% of the former (B20). ${ }^{7}$ Pure biodiesel is now being used for public transport in e.g., city buses in Sweden. ${ }^{8}$

Switching from petroleum diesel to biodiesel changes the "chemical" environment of plastic components and the effect of the fuel on the plastic component will be consequently different. ${ }^{9}$ Regardless of feedstock, biodiesel consists of fatty acid methyl esters, $^{3}$ the average carbon number being ca. 18 (varying with the type of feedstock), whereas petroleum diesel is made up of hydrocarbons with an average carbon number of 12 . The molecular differences between the fuels lead to differences in fuel solubility and diffusion behaviour in the polymer., ${ }^{4,5}$ In addition, biodiesel is susceptible to oxidation due to the presence of unsaturated carbon bonds, leading to the formation of aggressive species such as carboxylic acids and hydroperoxides. ${ }^{10,11}$ These oxidative products can have a strongly deteriorating effect on most of the materials used in fuel systems, ${ }^{1,10}$ the thermal instability of biodiesel may also lead to chemical reactions between biodiesel and polymer chains under high service temperatures. ${ }^{12,13}$ These interactions require a reassessment of both the short-term and long-term performance of polymer components in contact with biodiesel to achieve a problem-free fuel switch in vehicles. ${ }^{14-16}$

Polyamides (PAs) such as PA12 and PA6 are commonly used in vehicle fuel systems because of their good balance of mechanical properties and barrier properties against hydrocarbons, ${ }^{17-20}$ but the degradation of PAs in diesel, biodiesel, or mixtures of these has been scarcely investigated. Choudhury et al. ${ }^{21}$ reported that both the tensile strength and the elastic modulus of PA6 decreased by $20 \%$ after 1 -week exposure to biodiesel at room temperature. Nascimento et al. ${ }^{22}$ showed that the tensile strength and the strain-at-break of PA12, aged in biodiesel for $5000 \mathrm{~h}$ at $100{ }^{\circ} \mathrm{C}$, were only ca. $25 \%$, and $2 \%$ of that of unaged PA 12 , respectively. In our previous work, it was found that diesel/ biodiesel extracted the plasticizer from the inner PA6 layer of a PAbased multilayer fuel pipe, so that the pipe lost its flexibility. ${ }^{23}$ The inner PA6 layer was extensively oxidized after prolonged exposure, leading to a significant decrease in the toughness and extensibility of the pipe. ${ }^{23}$ The fuel diffusivity in the polymer is a factor that influences the kinetics of degradation, and can also lead to the leakage of fuel through the pipe system. Despite its importance, the diffusivity has not been determined for diesel and biodiesel, and mixtures of these, in common fuel pipe materials/ systems, perhaps because of the complexity of the fuel in that it contains a variety of molecules and that measurements have to be made at high temperatures.

In the present study, the ageing behaviour (effects on physicochemical and mechanical properties) of PA12 exposed to diesel, biodiesel and a commercial mixture of these (20 vol.\%

\footnotetext{
${ }^{1}$ Fibre and Polymer Technology, KTH Royal Institute of Technology, SE-100 44 Stockholm, Sweden; ${ }^{2}$ Polymer Centre, Volvo Car Corporation, SE-405 31 Göteborg, Sweden and ${ }^{3}$ Materials Technology, Scania CV AB, SE-151 87, Södertälje, Sweden

Correspondence: X-F. Wei (xinfengw@kth.se) or Mikael S. Hedenqvist (mikaelhe@kth.se)
}

Received: 24 October 2018 Accepted: 11 December 2018

Published online: 04 January 2019 

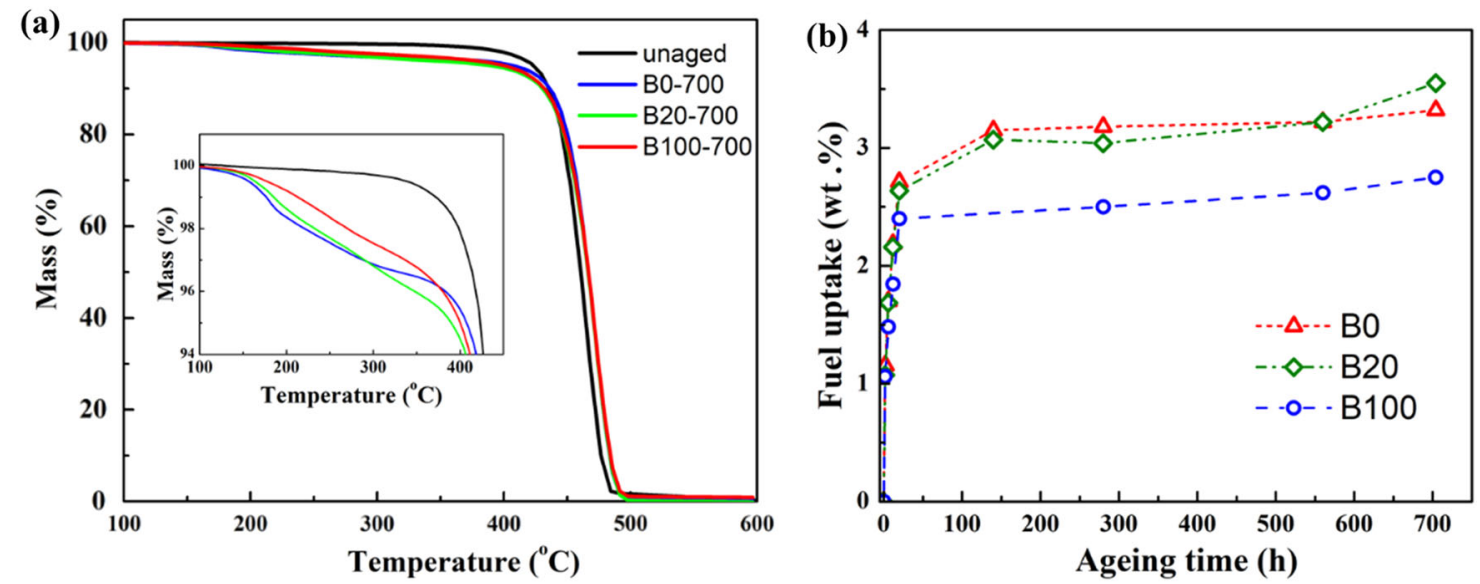

Fig. 1 a TG curves of the unaged PA12 sample and samples aged at $125^{\circ} \mathrm{C}$ for $700 \mathrm{~h}$ in B0, B20 and B100, and b fuel uptake of PA12 samples immersed in B0, B20 and B100, plotted as a function of ageing time. The inserted figure in a shows enlarged TG curves in the mass region from 94 to $100 \%$. The fuel uptake in b was determined as the initial mass loss obtained from TG curves at $320^{\circ} \mathrm{C}$
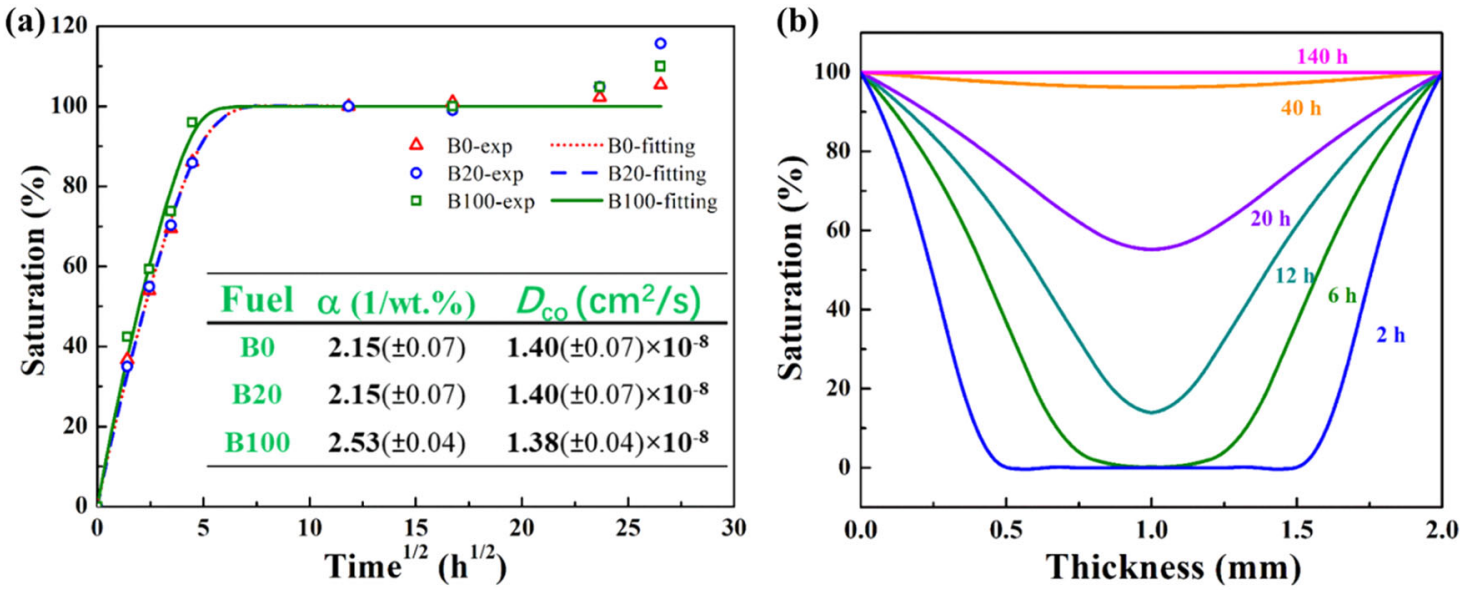

Fig. 2 a Normalized experimental and fitted mass uptake versus the square root of time of PA 12 samples exposed to $\mathrm{B} 0$, B20 or $\mathrm{B} 100$ at $125^{\circ} \mathrm{C}$; b fuel profiles of $\mathrm{B} 0$ generated with the diffusion model. The adjustable parameters determined from the fitting are listed in the table inserted in a

biodiesel) were investigated. The challenge was to test the material at a high temperature with conditions as close as possible to those in real vehicle/engine fuel systems. The fuel diffusivity, treating each fuel and fuel mixture as a single diffusing component, was also estimated.

\section{RESULTS AND DISCUSSION}

Fuel uptake

All the aged samples showed a mass loss between 100 and $320^{\circ} \mathrm{C}$ before the decomposition of the PA12, which started at ca. $450^{\circ} \mathrm{C}$ (Fig. 1a). This initial mass loss was ascribed to the evaporation of absorbed fuel and was consequently a measure of the fuel uptake. Figure $1 \mathrm{~b}$ shows that the fuel uptake increased rapidly in the early stage of exposure, and then levelled off. The values in the "plateau" region revealed that PA12 absorbed more B0 and B20 ( 3.2 wt.\%) than B100 ( 2.3 wt.\%), i.e., the solubility of petroleum diesel in PA12 was greater than that of biodiesel at $125^{\circ} \mathrm{C}$, and the addition of 20 vol.\% biodiesel to it did not change the level of uptake. The uptake kinetics in the early stages, until the plateau, could be described well with the present diffusion model using a concentration-dependent diffusivity (note the agreement between the experimental data and the curves fitted to the experimental data in Fig. 2a). Overall, the high-temperature kinetics were relatively similar for all three fuels (Fig. 2a). Although the zero-concentration diffusivity $\left(D_{\text {co }}\right)$, the diffusivity of a "hypothetical" un-plasticised material, was insignificantly different, the plasticization power $(a)$ was higher for B100 than for B0 and B20. Hence, the biodiesel molecules had a greater plasticizing effect on PA12 than those of petroleum diesel, which is in accordance with the glass transition temperature and mechanical data given below. Figure $2 \mathrm{~b}$ shows the time evolution of the concentration profiles of $\mathrm{B} 0$. The results of the plasticization of the PA12 by the fuel led to relatively steep concentration gradients during the uptake period (Fig. 2b), compared to the case if the diffusivity had been concentration-independent (not shown).

A second additional uptake was observed at longer exposure times for all fuels (after ca. 500 h) (Fig. 1b and Fig. 2a), which was probably an effect of the ageing-induced oxidation of the PA12 material (see below).

Extraction of monomers and oligomers from PA12

When, after ageing the PA12 specimens at $125^{\circ} \mathrm{C}$, the three fuels were cooled down to ambient temperature, they became turbid (illustrated for B100 in Fig. 3a). This was due to the precipitation of material extracted from the polymer and was indicated by the fact that it did not occur for the fuels aged without the PA12 specimens. All the characteristic IR-absorption bands of 
(a)

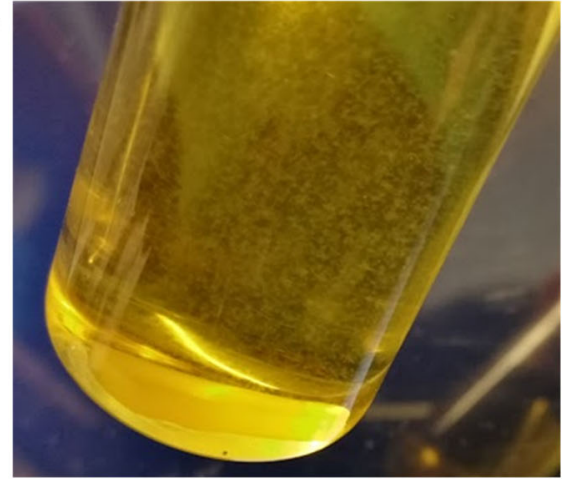

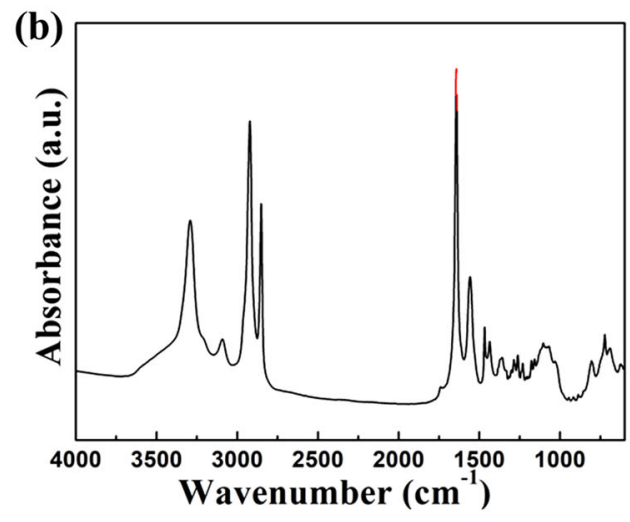

(c)

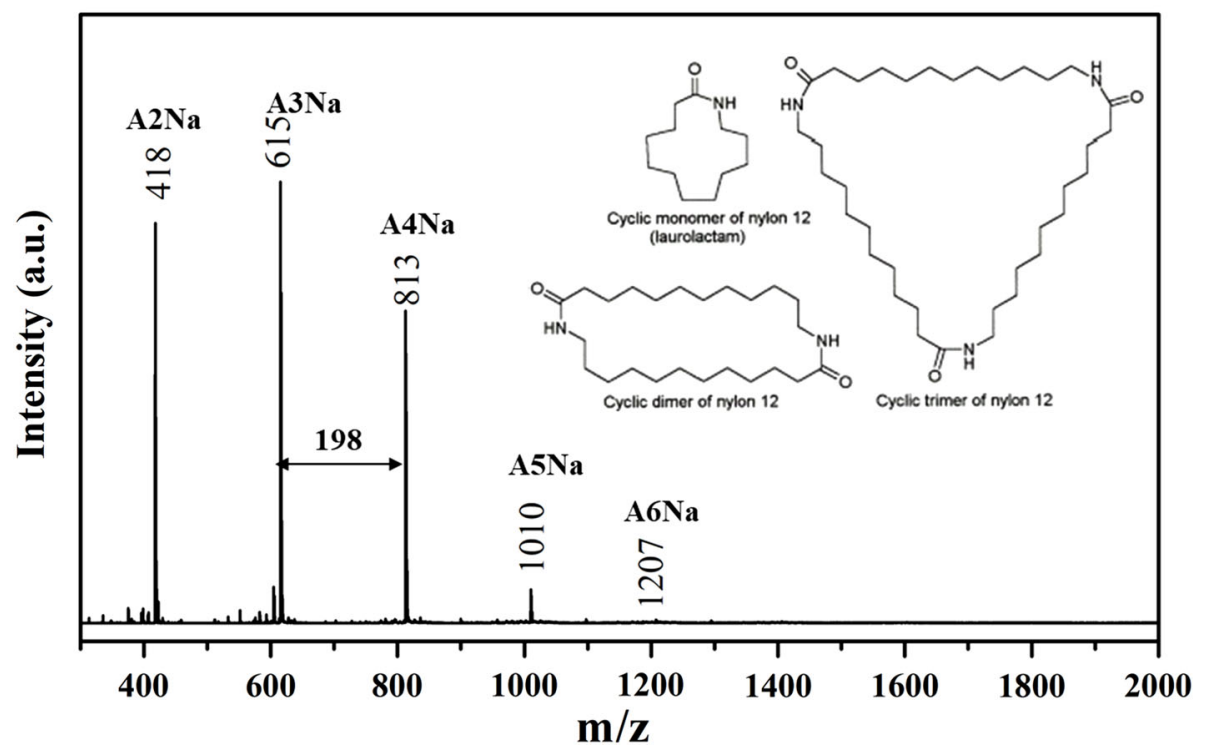

Fig. 3 a B100 in a sealed glass vial with a PA12 specimen after $48 \mathrm{~h}$ ageing at $125^{\circ} \mathrm{C} ; \mathbf{b}$ IR and c MALDI-TOF spectra of the insoluble material separated from the fuel present in a
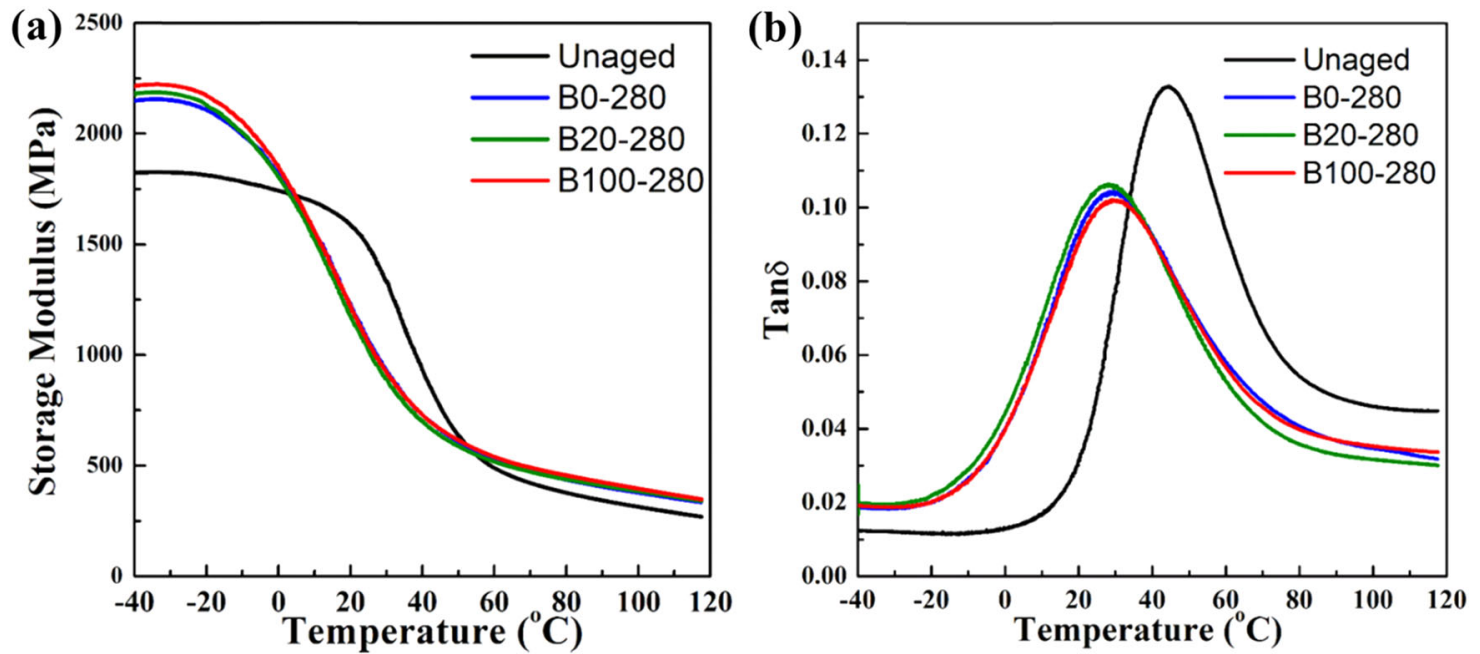

Fig. 4 a Storage modulus and $\mathbf{b}$ tan $\delta$ of the unaged sample and samples aged for $280 \mathrm{~h}$ in the different fuels

PAs (e.g., the amide I band at $1645 \mathrm{~cm}^{-1}$, amide II band at $1556 \mathrm{~cm}^{-1}$ and $\mathrm{N}-\mathrm{H}$ stretching band at $3290 \mathrm{~cm}^{-1}$ ) were observed in the IR spectrum of the precipitated material (Fig. $3 b)$, indicating that this consisted of PA12 material extracted by the fuel. The MALDI-TOF mass spectrum showed that the extracted fuel-insoluble material consisted of PA12 oligomers with a number of repeating units smaller than 7 (Fig. 3c). It was not possible to detect the PA12 monomers (with a size smaller than $\mathrm{m} / \mathrm{z}$ 198) by MALDI-TOF, due to its limitation on the low molar mass side. PA12 commonly contains both monomers and 

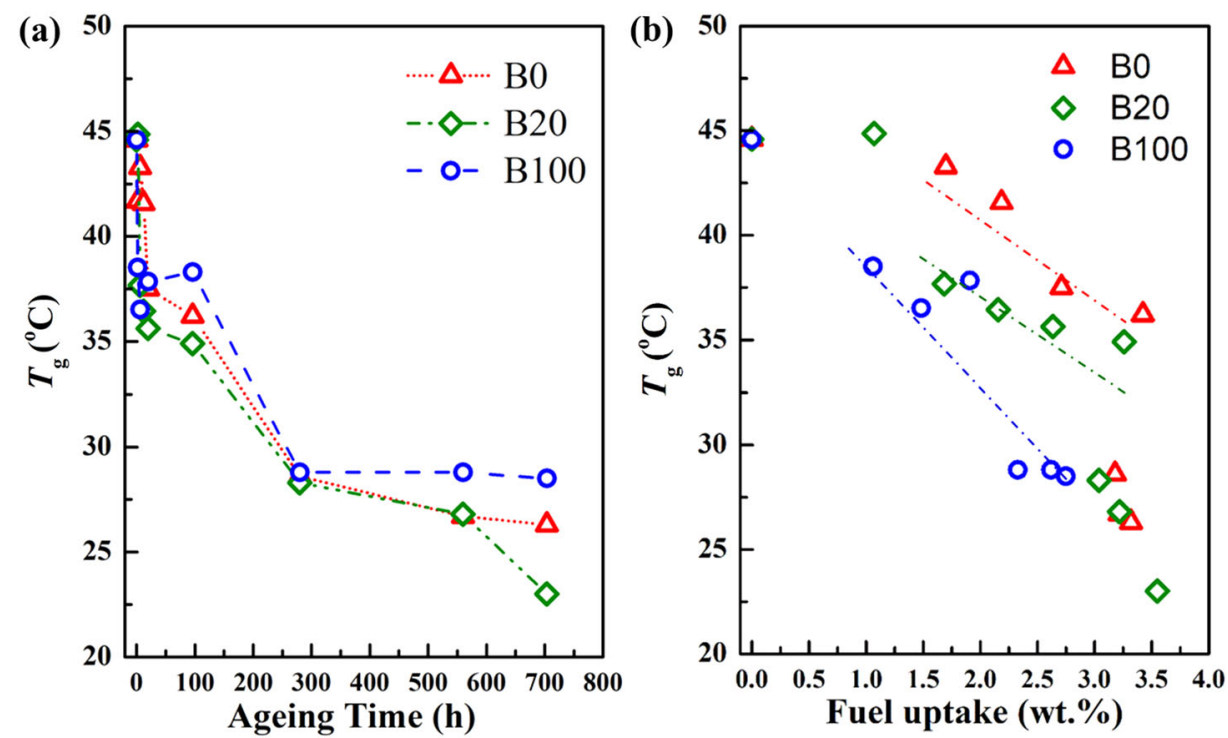

Fig. $5 T_{\mathrm{g}}$ plotted as functions of $\mathbf{a}$ ageing time and $\mathbf{b}$ fuel uptake, respectively. The broken lines in $\mathbf{b}$ highlight the trend in data where the fuel uptake is between 1 and $3 \mathrm{wt} . \%$ (short exposure time)
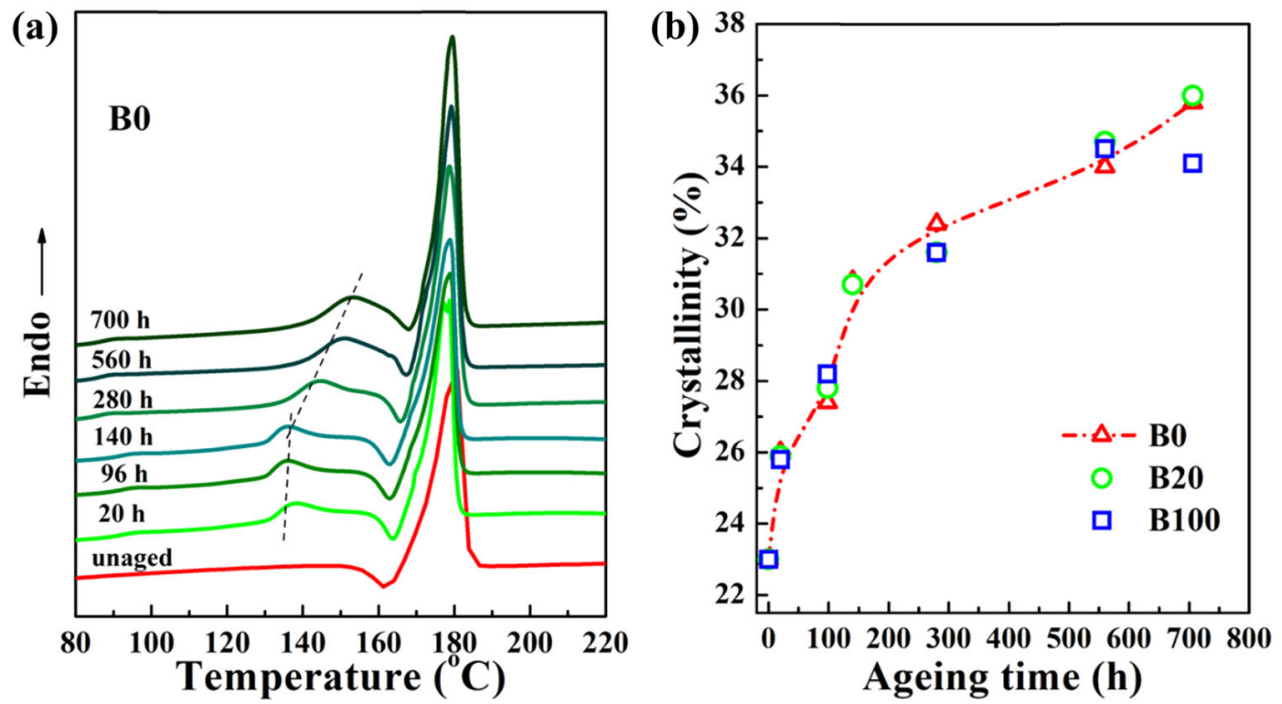

Fig. 6 a DSC heating curves of the unaged sample and samples aged in B0 and b crystallinity of PA12 samples plotted as a function of ageing time

oligomers, ${ }^{24}$ so it is reasonable to assume that monomers were also extracted on the exposure to fuel. On the other hand, oligomers with a number of repeating units greater than 6 were not extracted, because of size effects (molecular entanglements and a larger number of hydrogen bonds per molecule). The extracted material in the first $48 \mathrm{~h}$ exposure accounted for ca. 0.2 wt.\% of the mass of the immersed PA12 specimen. The extraction of PA12 monomers and oligomers to the fuel is known to cause precipitation and injector/filter clogging during service and is one of the main side effects when PAs are used as fuel-contacting material in vehicle fuel systems. ${ }^{25}$ It should be noted that the extraction of monomers and oligomers occurred here in all three types of fuel.

Change in glass transition temperature

Figure 4 shows that the decrease in the storage modulus and the increase in $\tan (\delta)$ of the $280 \mathrm{~h}$-aged samples started at $-20^{\circ} \mathrm{C}$, which was a lower temperature than that observed for the unaged sample $\left(\mathrm{ca} .0^{\circ} \mathrm{C}\right)$. The $\tan (\delta)$ peak for the aged samples appeared at $28^{\circ} \mathrm{C}$, which was $17^{\circ} \mathrm{C}$ lower than for the unexposed sample $\left(45^{\circ} \mathrm{C}\right.$ ). A clear plasticization (decrease in $T_{\mathrm{g}}$ ) of the PA12 material was then observed for all three fuels, in line with the diffusivity data given above (none zero a value).

Figure $5 \mathrm{a}$ shows that the decrease in $T_{\mathrm{g}}$ was largest during the first hours of testing and then gradually levelled off. After $280 \mathrm{~h}$, the glass transition had dropped to $28^{\circ} \mathrm{C}$ for B100 and $26^{\circ} \mathrm{C}$ for B0 and B20. A further decrease in $T_{\mathrm{g}}$ with longer ageing times was observed partially with B20, but also to some extent with B0. The lower reduction in $T_{\mathrm{g}}$ in $\mathrm{B} 100$ was due to its lower uptake in PA12 compared to that of the other fuels. Figure $5 \mathrm{~b}$ shows that in the early stage of ageing, the decrease in $T_{\mathrm{g}}$ was fastest in B100, and slowest in B0, which indicates that biodiesel had a stronger plasticizing effect on the PA12 material than petroleum diesel. 

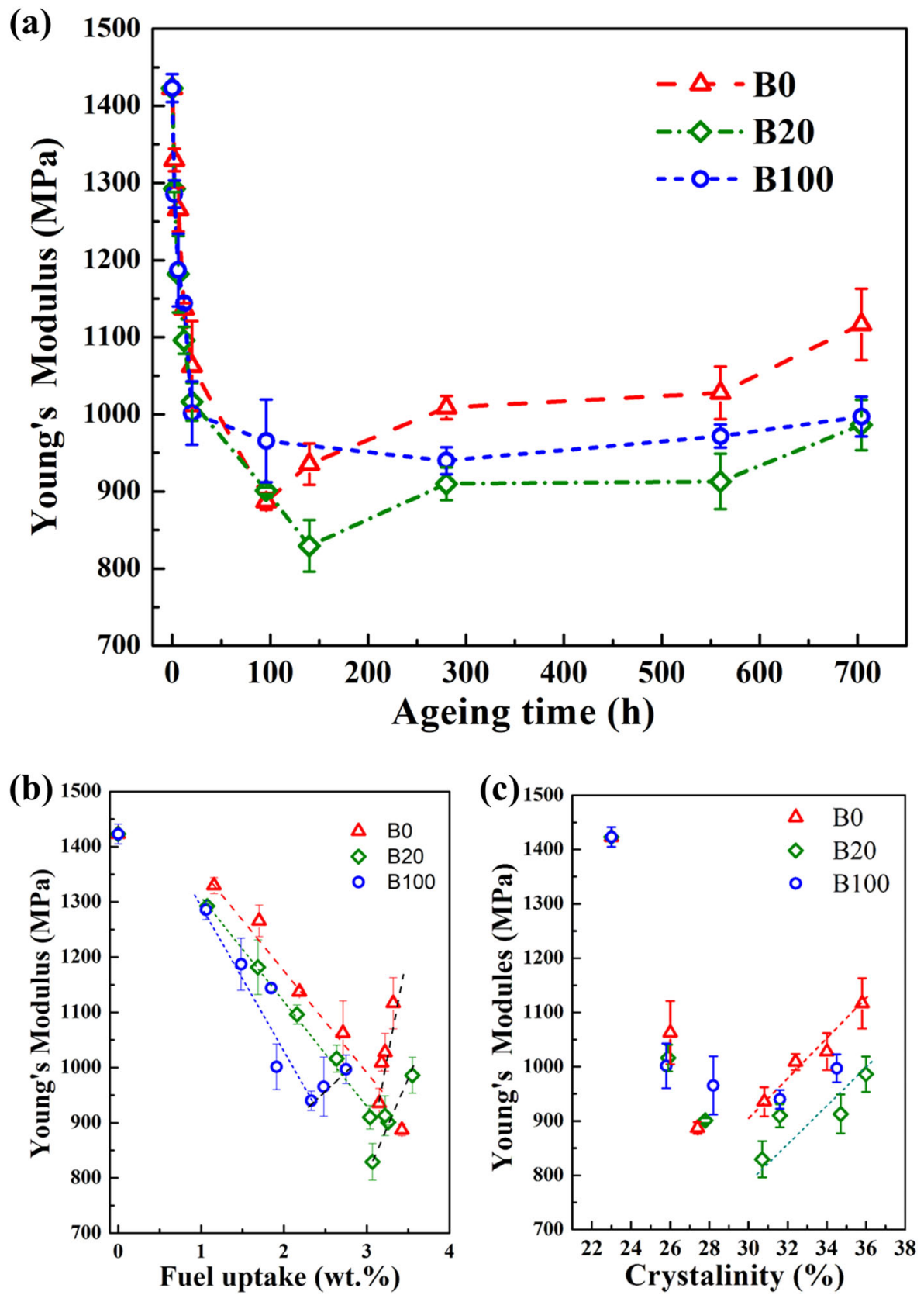

Fig. 7 Young's modulus plotted as functions of $\mathbf{a}$ ageing time, $\mathbf{b}$ fuel uptake and $\mathbf{c}$ crystallinity. The broken lines in $\mathbf{b}$ and $\mathbf{c}$ show the trends in the corresponding regions. The error bars were taken as the standard deviation for Young's modulus

This finding is in good agreement with the plasticizing power obtained from the diffusivity measurements ( $a$, Fig. 2a).

Annealing effect

The unaged PA12 sample had a single melting peak at $180^{\circ} \mathrm{C}$ (Fig. 6a). After ageing, a new small melting peak appeared at $135^{\circ} \mathrm{C}$, which shifted towards higher temperatures and became larger with increasing ageing time. It is known that when crystallizable polymers are annealed at elevated temperatures, a new melting peak appears $\mathrm{ca} .10^{\circ} \mathrm{C}$ above the annealing temperature, which shifts to a higher temperature linearly with increasing annealing time. ${ }^{26-28}$ This is due to the formation of new thinner crystals that grow in thickness with increasing annealing time. Thus, the new peak at $135^{\circ} \mathrm{C}$, only $10^{\circ} \mathrm{C}$ above the $125^{\circ} \mathrm{C}$ ageing temperature, was assigned to the melting of 'new' crystals formed during the annealing/ageing at $125^{\circ} \mathrm{C}$. Due to the annealing, the crystallinity increased significantly by $9 \%$, from 23 to $32 \%$, during the first $280 \mathrm{~h}$ ageing, and then slowly approached $36 \%$ after $700 \mathrm{~h}$ ageing (Fig. 6b). It is noteworthy that the annealing-induced increase in crystallinity was essentially independent of the fuel type (Fig. 6b). It should also be noted that any oxidation-induced chain scission did not lead to any observable increase in crystal size, as indicated by the unchanged position of the main melting peak during ageing (Fig. 6a).

\section{Stiffness}

Figure 7a shows that the Young's modulus of the samples aged in fuels displayed a two-stage behaviour with increasing ageing time: (i) a fast and large decrease followed by (ii) a slow increase. The modulus of the samples exposed to B0, B20 and B100 

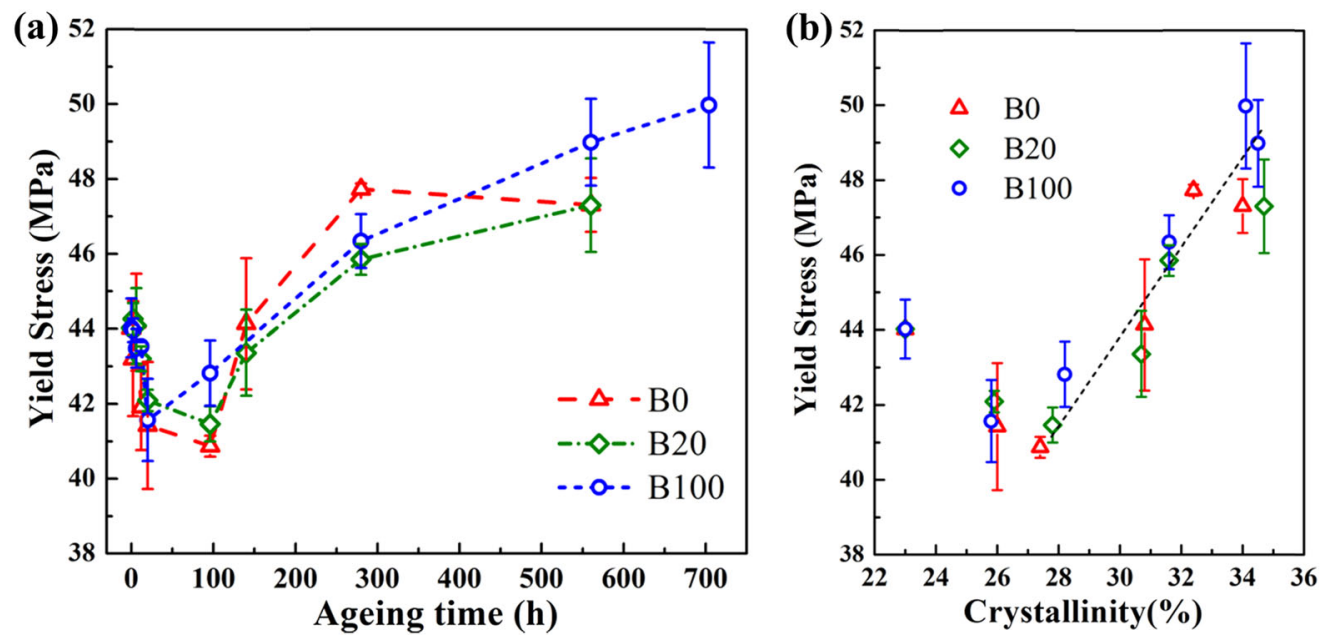

Fig. 8 Yield strength plotted as a function of $\mathbf{a}$ ageing time and $\mathbf{b}$ crystallinity, respectively. The error bars were taken as the standard deviation for yield strength
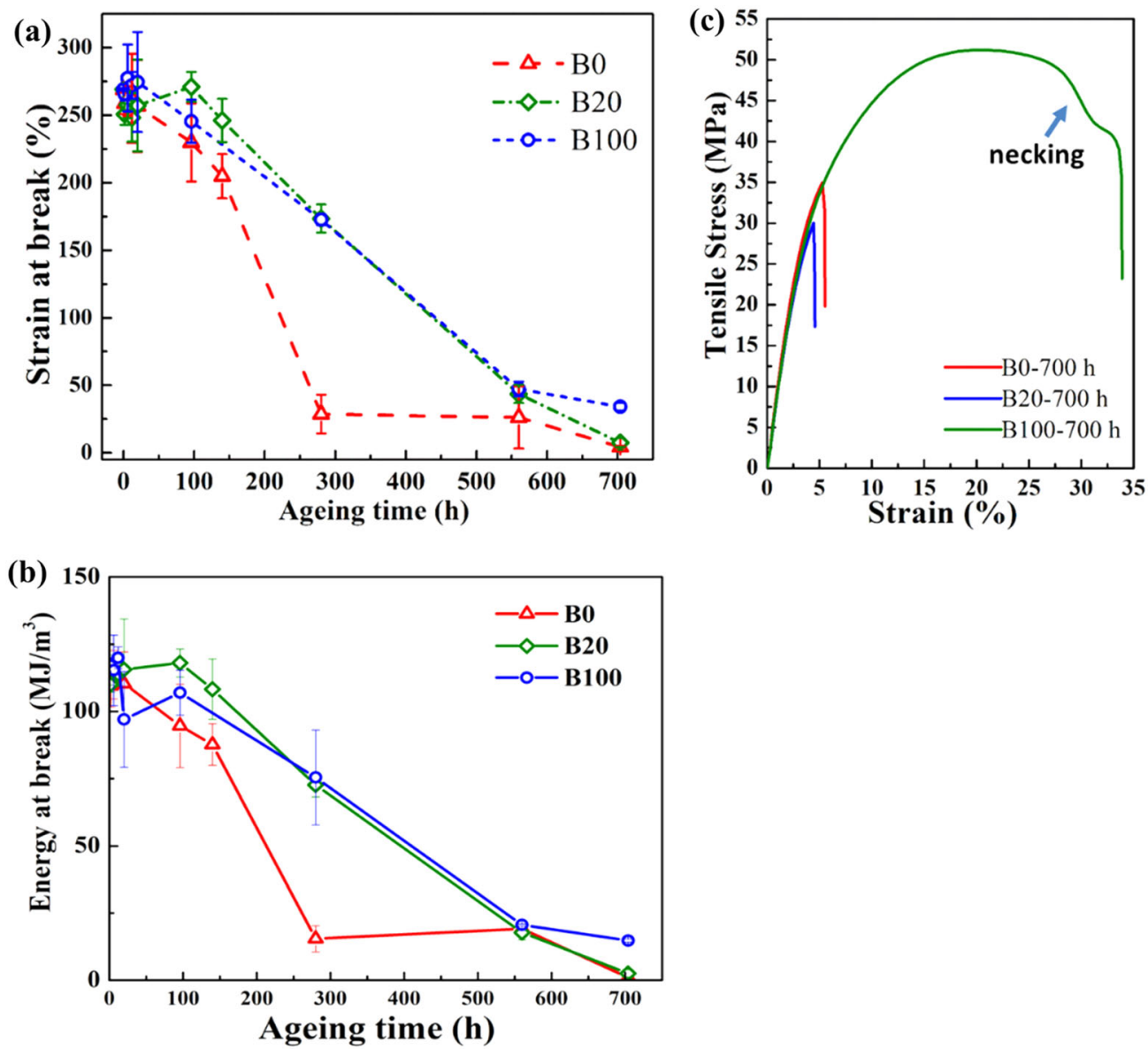

Fig. 9 a Strain-at-break and $\mathbf{b}$ energy-at-break plotted as a function of ageing time and $\mathbf{c}$ stress-strain curves of the samples aged for $700 \mathrm{~h}$ in different fuels. The error bars were taken as the standard deviation

decreased by a maximum of 38,41 , and $33 \%$, respectively. The reason for the two-stage behaviour is shown in Fig. 7b, c. The modulus decreased linearly with increasing fuel uptake (Fig. $7 \mathrm{~b}$ and initial stage in Fig. 7a) due to the plasticizing effect of the fuel, which was greatest for the biodiesel (steepest slope of modulus vs. fuel uptake in Fig. 7b). The fuel diffusivity, glass transition temperature and mechanical data thus all show that the plasticizing effect of biodiesel is greater than that of petroleum diesel. The greatest decrease in modulus was observed for the sample exposed to B20, since B20 was the fuel that was absorbed the most (3.2 wt.\%). When the saturation of fuel uptake was reached, the modulus increased slowly with ageing time after the 

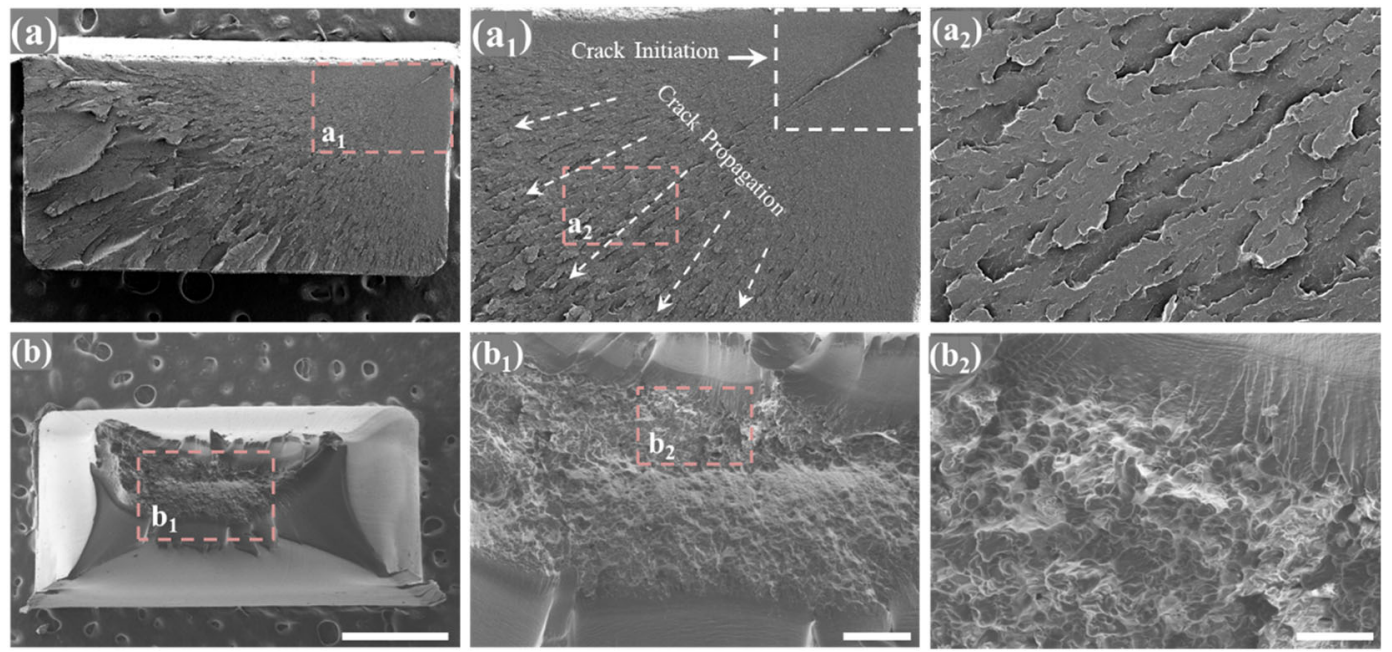

Fig. 10 SEM images of the tensile fracture surfaces of the samples aged for $700 \mathrm{~h}$ in $\mathbf{a} \mathrm{B} 0$ and $\mathbf{b} \mathrm{B} 100$. The length of scale bars from left to right is $1000,200,50 \mu \mathrm{m}$, respectively
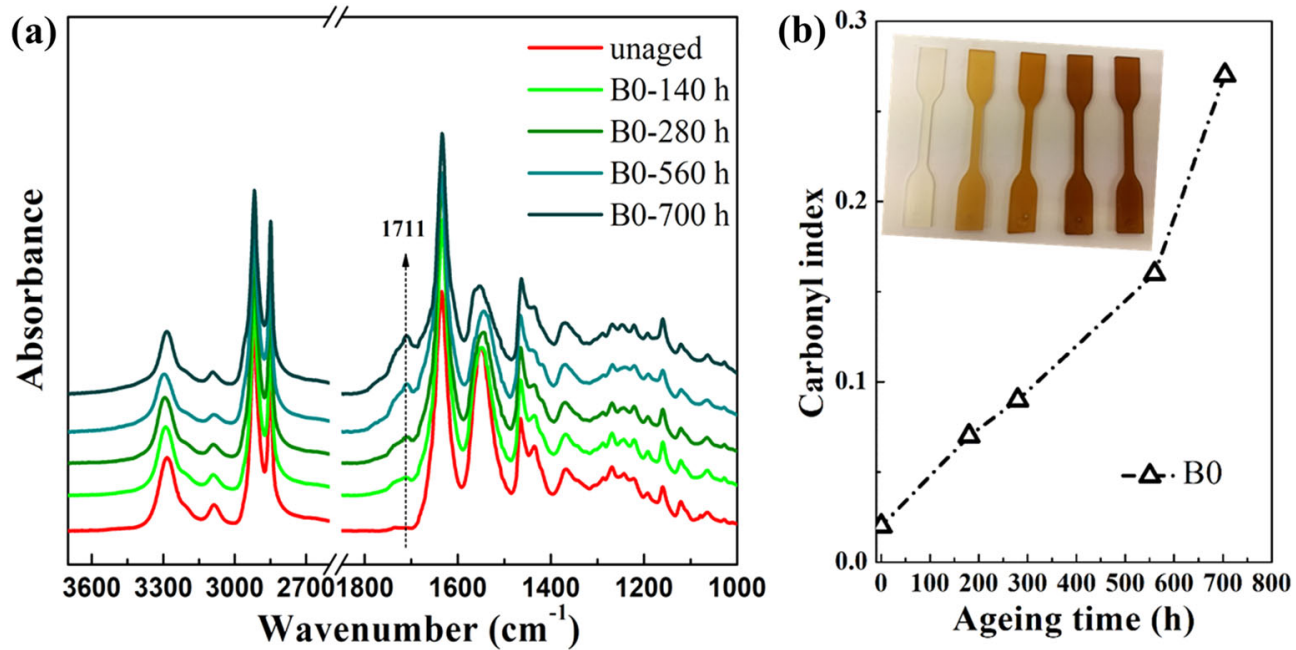

Fig. 11 a IR spectra of the surface of the unaged sample and samples aged in B0 and $\mathbf{b}$ the carbonyl index plotted as a function of ageing time. The inset picture in b shows the unaged sample (to the left) and samples aged in B0 for 140, 280, 560, and 700 h (from left to right)

initial regional loss on the modulus (Fig. 7a and data set on the right part in Fig. 7b). This is explained as being due to an ageing/ annealing-induced increase in crystallinity (dataset in the righthand part of Fig. 7c).

\section{Tensile strength}

Figure 8a shows that the yield strength first decreased (fuelinduced plasticization/weakening) and then increased with increasing ageing time, and Fig. $8 \mathrm{~b}$ shows that the increase in yield strength was due to the increase in crystallinity. The effect of the increase in crystallinity was significantly greater on the strength than on the modulus (cf. Fig. 7a, c with Fig. 8a, b). In fact, the PA12 samples aged for more than $140 \mathrm{~h}$ were both stronger (yield strength) and softer (modulus) than the unaged material.

Embrittlement of PA12 aged in fuels

Figure 9a shows that, when considering the full ageing period, the ductility/strain-at-break decreased significantly in all the fuels. The decrease in toughness (energy-at-break) showed the same trend (Fig. 9b). The decrease in both ductility and toughness beyond
$140 \mathrm{~h}$ was however much faster in the samples exposed to diesel (B0) than in those exposed to B100 or B20. After $700 \mathrm{~h}$, the samples aged in B0 and B20 were brittle and broke before the yield point, but the sample exposed to B100 still exhibited yielding/necking before fracture (Fig. 9c). This difference was also evident in the SEM images of the fracture surfaces, illustrated in Fig. 10. The sample aged in BO for $700 \mathrm{~h}$ showed a feature-less smooth typical brittle fracture surface, whereas the fracture surface of the sample aged in B100 showed signs of significant plastic deformation. One reason for this observed difference in the embrittlement kinetics between the fuels is discussed in a later section below.

The loss of extensibility/ductility of PAs is basically due to oxidation which induces chain scission and chemicrystallization. $^{29-32}$ When exposed to B0 at $125^{\circ} \mathrm{C}$, the PA12 samples gradually changed colour from white (unaged) to yellow after $140 \mathrm{~h}$ and to yellowish brown after $560 \mathrm{~h}$ (inset in Fig. $11 \mathrm{~b})$, indicative of oxidation. The oxidation was confirmed by the appearance of a carbonyl peak at $1711 \mathrm{~cm}^{-1}$ in the IR spectra of the aged samples (Fig. 11a), and the degree of oxidation increased with increasing ageing time (Fig. 11b). 
(a)

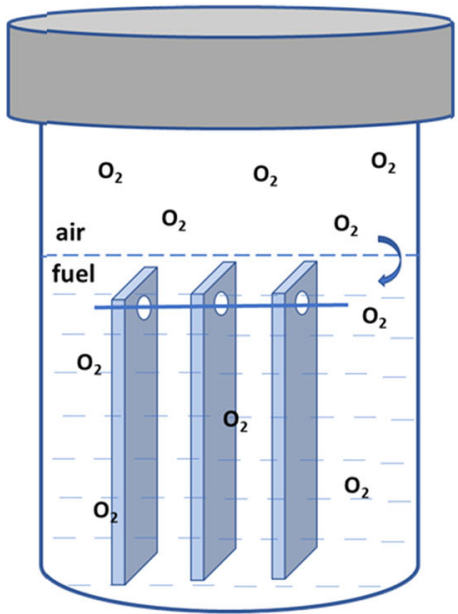

Volume Ratio: air/fuel=1:2

(b)

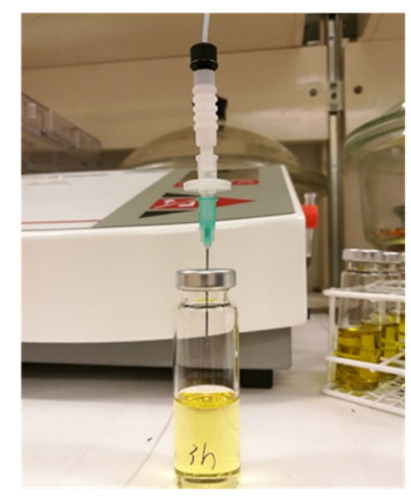

(c)
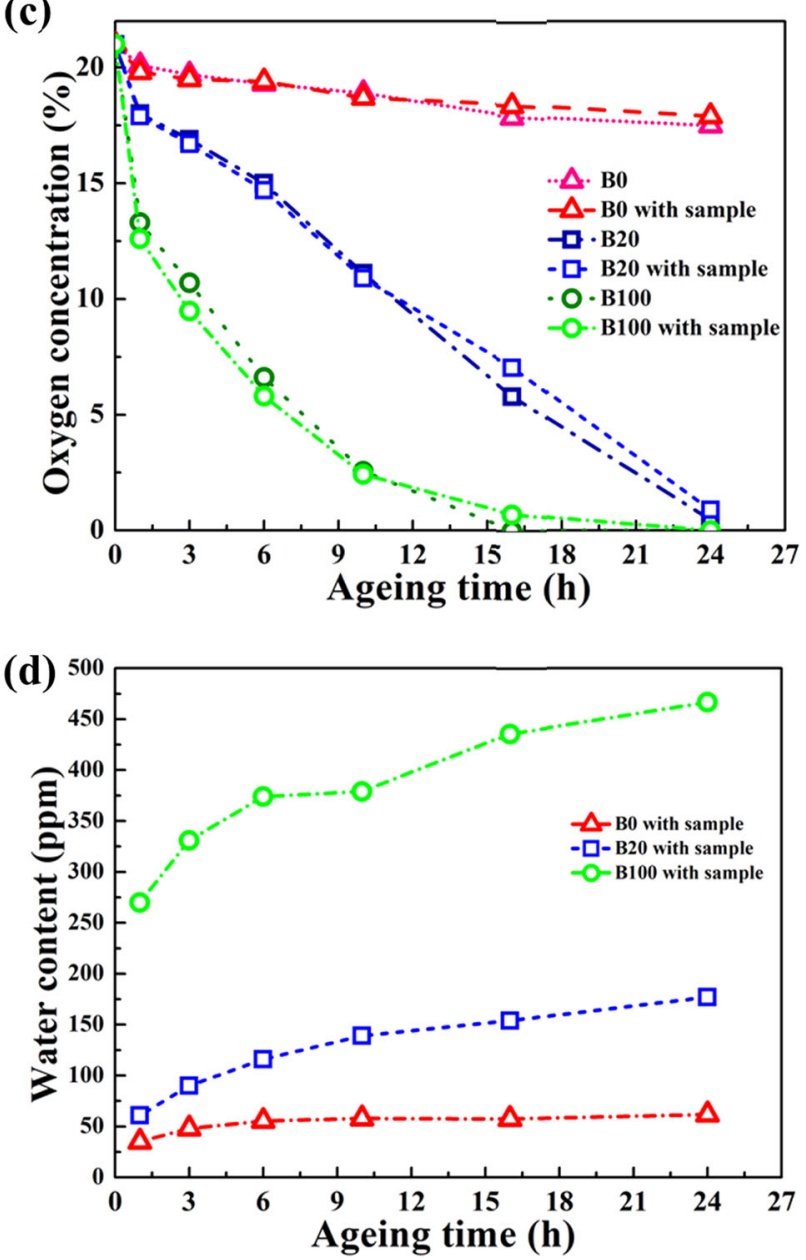

Fig. 12 a Schematic figure of the oxygen condition in autoclaves; $\mathbf{b}$ image of the set-up used for oxygen concentration measurement in the gas phase in the sealed glass vials; $\mathbf{c}$ the oxygen concentration in the gas phase and $\mathbf{d}$ water content in the fuels

Yellowing was also observed in the samples aged in B20 and B100, but it was not possible to reveal the degree of oxidation quantitatively with IR since the sorbed fuels could not be completely extracted from the samples (revealed by the early mass loss in the TG curves of the extracted samples) and the ester group on the biodiesel molecules contributed to the carbonyl band. Akhlaghi et al. ${ }^{12}$ also observed unextractable biodiesel in fluoro-elastomers after exposure to $\mathrm{B} 100$ at $100^{\circ} \mathrm{C}$.

Since the testing temperature was higher than the flash points of both $\mathrm{BO}$ and $\mathrm{B} 20$, the samples were for safety reasons aged in a closed environment, except during the replacement of fuel, which means that the amount of oxygen available in the autoclaves was limited. To obtain more information about the PA12 oxidation and its relation to the available oxygen, the kinetics of oxygen consumption in the autoclaves during the ageing at $125^{\circ} \mathrm{C}$ was assessed by measuring the change in oxygen concentration in the head (gas) space in a small-scale sealed glass vial with the same ratio of head space to liquid volume as in the autoclave (Fig. 12a, b). Figure $12 \mathrm{c}$ shows that during ageing, the oxygen concentration in the head space remained almost unchanged at $20 \%$ in the sealed vials filled with $\mathrm{B} 0$ but decreased rapidly with $\mathrm{B} 20$ and $\mathrm{B} 100$. The head space was devoid of oxygen in the B20 and B100 cases after 15 and $24 \mathrm{~h}$, respectively. Figure $12 \mathrm{~d}$ shows that the water content in B20 and B100 increased during ageing. Since water is a product of biodiesel oxidation, this increase in water content indicated that the biodiesel in B20 and B100 was oxidized at $125^{\circ} \mathrm{C}$. Furthermore, the decrease in oxygen concentration in the vials was the same with and without a PA12 sample immersed in the fuel (Fig. 12c), indicating that the oxygen was consumed mainly by oxidation of the biodiesel and not by the polymer.

Due to the rapid oxygen consumption by the biodiesel, the PA12 samples immersed in B20 and B100 were exposed to a lower concentration of oxygen than the sample immersed in B0. This shortage of oxygen reduced the rate of oxidation of the polymer and slowed down the embrittlement process in B20 and B100 (cf. the strain-at-break values at $280 \mathrm{~h}$ in Fig. 9a). Hence, only in the case of B0 did the oxygen supply in the autoclave resemble the real case in a vehicle fuel system, where oxygen is continuously available. This shows the difficulty of testing polymers exposed to highly oxidation-sensitive fuels at elevated temperatures (above the flash point of the fuels) under 'real' vehicle conditions.

The ageing of PA12, mimicking high-temperature conditions (long-term driving at high speed) in a vehicle fuel line system, exposed to petroleum diesel, biodiesel or a mixture of these, was investigated. PA12 experienced essentially the same type of ageing mechanisms, although to different degrees, in all three fuels; fuel uptake and fuel-induced plasticization, fuel-induced extraction of monomers and oligomers, oxidation and annealinginduced crystallization. The biodiesel had a lower solubility but a slightly faster uptake than petroleum diesel in PA12. The faster uptake of biodiesel was due to its stronger plasticizing effect on PA12, indicated by its larger plasticization power $(a)$ and greater 
depression of the glass-transition temperature. The fuel uptake and annealing had an effect on both the modulus and tensile strength. The fuel-induced plasticization dominated in the early stage of ageing, leading to a large decrease in stiffness, while the annealing-induced increase in crystallinity led to an increase in modulus during the later stages of ageing. The PA12 ductility and toughness decreased during ageing, and the change in the biodiesel fuels (B100 and B20) was slower than in the petroleum diesel (B0). This apparent difference was a consequence of the limited availability of oxygen in the autoclave in the presence of the highly oxidation-sensitive biodiesel. This shows that there is a challenge to test safely under 'real' vehicle conditions under extreme driving conditions (high temperature) in the case of highly oxidation-sensitive fuels.

The present results indicate that the high-temperature interactions between the fuel and the polymer, and the associated ageing mechanisms, are 'generic' in the sense that they are expected to occur, although to various extents, not only with PA12 but also with many other polymers. The results show that care must be taken when selecting a polymer for an application in a vehicle where it will be exposed to fuels at high temperature. More specifically, it is shown that PA12 single layer materials are problematic to use under the conditions presented here.

\section{METHODS}

\section{Materials and fuels}

Injection-moulded $2 \mathrm{~mm}$ thick dumbbell bars (type $5 \mathrm{~A}$, ISO527-2-5A) of stabilized PA12, with a trademark of VESTAMID L1940 and a density of $1010 \mathrm{~kg} / \mathrm{m}^{3}$, were kindly supplied by Evonik Industries AG, Germany. The width and length of the narrow region and the total length of the dumbbell specimens were 4,26 and $86 \mathrm{~mm}$, respectively. This PA12 grade is commonly used in vehicle fuel systems. The samples were aged in three types of fuel: petroleum diesel (B0, Swedish environmental class 1 diesel (MK1)), biodiesel (B100, a commercial rapeseed biodiesel), and B20, which is a mixture of MK1 and B100 (20 vol.\% biodiesel). The flash points of diesel and biodiesel are 56 and $101{ }^{\circ} \mathrm{C}$, respectively. All the fuels were delivered by Preem AB, Sweden.

\section{Ageing in autoclaves}

The as-received samples of PA 12 were dried at $80^{\circ} \mathrm{C}$ in a vacuum oven for $24 \mathrm{~h}$ and then stored in a desiccator with silica gel before ageing. The dry samples were aged in B0, B20 and B100 at $125^{\circ} \mathrm{C}$ in three specially built $2.3 \mathrm{~L}$ sealed autoclaves with a diameter of $12 \mathrm{~cm}$. Sealed autoclaves were used for safety reasons, the flash points of the fuels being lower than the ageing temperature. A small hole was drilled in the broad part of the dumbbell specimen, and 6 specimens were strung together into a single group with a steel wire. To avoid direct contact between neighbouring specimens during exposure to the fuels, a short Teflon washer was strung on the wire between each specimen. The prepared samples were placed in three autoclaves where they were immersed in B0, B20 and B100, respectively. The volume of fuel was in each case twice the size of the head space (air) in the sealed autoclaves. The autoclaves were placed in an oven at $125 \pm 1{ }^{\circ} \mathrm{C}$, and the aged fuel was replaced by fresh fuel after every two days to mimic the refuelling process. In addition, three smaller autoclaves $(1 \mathrm{~L}$, diameter: $10 \mathrm{~cm}$ ) were used for ageing the samples in fuel in a shortterm (less than $100 \mathrm{~h}$ ) ageing. Samples were taken from the autoclaves after different ageing times and stored in sealed polyethene plastic bags in a refrigerator (at a constant temperature of $4^{\circ} \mathrm{C}$ ) until further testing.

\section{Ageing in sealed glass vials}

In order to easily track the oxygen condition and fuel oxidation in the autoclaves during the ageing period, a small-scale ageing was carried out in sealed glass vials under the same conditions as in the autoclaves. $13 \mathrm{~mL}$ of fuel and a small piece of PA12 (ca. $20 \times 20 \times 2 \mathrm{~mm}^{3}$, cut from the dumbbell bar) were placed in a $20 \mathrm{~mL}$ headspace crimp vial, and the glass vial was then sealed with aluminium crimp caps (with a central hole of $10 \mathrm{~mm}$ in diameter having a butyl/PTFE septum). The volume ratio of the fuel to head-space (air) in the sealed vial was 2, the same as in the autoclaves. For comparison, sealed vials containing only fuel (no PA12) were also prepared. The prepared vials were placed in a ventilated oven at $125 \pm 1{ }^{\circ} \mathrm{C}$ for up to 2 days.

Determination of the composition of insoluble substances, the oxygen concentration of the gas phase and the water content in the fuels in the sealed glass vials

During ageing in the presence of the PA12 samples, insoluble material appeared in the fuels, and these were separated from the fuels by centrifugation (4000 rpm,10 min). The supernatants (fuels) were discarded, and the insoluble pellets were washed with ethanol (96 vol.\%, VWR International, Sweden) under stirring. The ethanol suspension obtained was then centrifuged and the supernatant was discarded. These cleaning steps were repeated three times and the insoluble substance was finally dried in an oven at $80^{\circ} \mathrm{C}$.

Matrix-assisted laser desorption/ionization time-of-flight (MALDI-TOF) spectra of the dried insoluble substances were acquired using an UltraFlex MALDI-TOF with SCOUT-MTP Ion Source (Bruker Daltonics, Bremen, Germany) equipped with a nitrogen laser $(337 \mathrm{~nm})$, a gridless ion source and a reflector. Dried-droplet samples were prepared by spotting $5 \mu \mathrm{L}$ of 2,5-dihydroxybenzoic acid (DHB) matrix solution $\left(15 \mathrm{mg} \mathrm{mL}^{-1}\right.$ in methanol), $1 \mu \mathrm{L}$ of the solution of insoluble substances $\left(5 \mathrm{mg} \mathrm{mL}^{-1}\right.$ in trifluoroacetic acid (TFA)), and $1 \mathrm{uL}$ of counter-ion solution of sodium trifluoroacetate ( $5 \mathrm{mg} \mathrm{mL}^{-1}$ in methanol) in a sandwich manner on a target plate. The spectra obtained were analysed with FlexAnalysis version 2.2 from Bruker Daltonics.

The oxygen concentration in the head-space in the sealed vials was measured by a PBI Dansensor CheckMate 9900 Oxygen Analyzer equipped with a probe with a needle holder and sampling hose. The water content in the fuel was determined in a Karl-Fischer titrator (831 KF coulometer, Metrohm, Switzerland)

\section{Thermogravimetry (TG)}

The fuel uptake by the PA12 samples was measured in a TG/DSC 1 (Mettler-Toledo, Switzerland). Specimens weighing ca. $7 \mathrm{mg}$, cut evenly in the direction perpendicular to the length direction of the specimens so as to represent the cross-section of the narrow region of the dumbbell specimen, were placed in a $70 \mu \mathrm{L}$ alumina crucible. TG curves were obtained at $25-600^{\circ} \mathrm{C}$ with a heating rate of $10^{\circ} \mathrm{C} \mathrm{min}-1$ and a nitrogen gas flow rate of $50 \mathrm{ml} \mathrm{min}^{-1}$.

\section{Dynamic mechanical analysis (DMA)}

To explore the changes in the glass transition temperature during ageing, unaged and aged samples were studied in a dynamic mechanical analyzer (Q800, TA Instruments, USA) with a three-point bending mode at an amplitude of $30 \mu \mathrm{m}$ and a frequency of $1 \mathrm{~Hz}$. The samples were heated from -40 to $140^{\circ} \mathrm{C}$ at a rate of $3^{\circ} \mathrm{C} \mathrm{min}{ }^{-1}$. The evaporation of absorbed fuel in this temperature range investigated is very low (based on the TGA results) and could be ignored.

\section{Differential scanning calorimetry (DSC)}

The melting behaviour was studied with a Mettler-Toledo DSC 1. Samples weighing ca. $5 \mathrm{mg}$, were placed in $40 \mu \mathrm{L}$ aluminium cups. The samples were heated from 20 to $250{ }^{\circ} \mathrm{C}$ at a rate of $10^{\circ} \mathrm{C} \mathrm{min}^{-1}$ in nitrogen with a gas flow rate of $50 \mathrm{~mL} \mathrm{~min}^{-1}$. The degree of crystallinity was calculated by dividing the melting enthalpy by the melting enthalpy of $100 \%$ crystalline PA12 $(209 \mathrm{~J} / \mathrm{g}){ }^{33,34}$ Before the DSC tests, small slices were cut from the samples and the sorbed fuel was extracted from the aged samples at $40 \pm$ $1{ }^{\circ} \mathrm{C}$ under ultrasonication for $30 \mathrm{~min}$ in acetone ( $\geq 99.8 \mathrm{wt} \%$; VWR International, Sweden) and for $30 \mathrm{~min}$ in n-hexane ( $\geq 99 \mathrm{wt} \%$; VWR International, Sweden). The extracted samples were finally dried at $80 \pm$ $1{ }^{\circ} \mathrm{C}$ for $48 \mathrm{~h}$ in a vacuum oven. A low content of fuel $(<1 \mathrm{wt} . \%$, determined by TG experiments) remained in the dry extracted samples, but their influence on the melting behaviour and crystallinity calculation was very small and hence safely ignored.

\section{Tensile tests}

Tensile tests were carried out at $23 \pm 1{ }^{\circ} \mathrm{C}$ and $50 \pm 2.5 \% \mathrm{RH}$ in an Instron 5944 Universal Tensile Testing Machine with a $10 \mathrm{kN}$ load cell, according to ASTM D638M. The specimens, with a gauge length of $40 \mathrm{~mm}$, were strained at a crosshead speed of $50 \mathrm{~mm} \mathrm{~min}^{-1}$. At least three replicates were performed for each sample. 
Scanning electron microscopy (SEM)

The morphology of the fractured surfaces of the tensile samples was examined in a field-emission scanning electron microscope (Hitachi S4800). Before the SEM analysis, the samples were coated with palladium using an Agar high-resolution sputter coater, model 208RH.

\section{Attenuated total reflectance infrared (ATR-IR) spectroscopy}

The IR spectra were recorded in a Perkin-Elmer Spotlight 400 equipped with a single ATR accessory (Golden Gate) from Graseby Specac, UK. The extracted samples described in the differential scanning calorimetry section were used for the measurements. The carbonyl index $(C l)$ of PA12 was determined as the ratio of the integrated oxidation band region between 1695 and $1760 \mathrm{~cm}^{-1}$ to the reference band peaking at $1460 \mathrm{~cm}^{-1}$ (1488-1397 $\mathrm{cm}^{-1}$, amide III band). The size of the latter is insensitive to oxidation. $^{30,35}$

\section{Fuel uptake kinetics}

The fuel mass uptake obtained with TG was modelled with Fick's second law $^{36}$ for a two-dimensional case (the cross-section of the narrow part of the dumbbell specimen $\left.\left(4 \times 2 \mathrm{~mm}^{2}\right)\right)$, using a diffusion coefficient that was dependent on the fuel concentration: ${ }^{37,38}$

$$
D=D_{c o} e^{a c}
$$

where $D_{\mathrm{co}}$ is the 'zero-concentration' fuel diffusion coefficient and $a$ is the fuel plasticizing power. The integrated concentration profiles, yielding fuel mass-uptake-versus-time curves and the fitting of these to the corresponding experimental uptake data, using $a$ and $D_{\mathrm{c} 0}$ as adjustable parameters, were all done in Comsol Multiphysics.

\section{DATA AVAILABILITY}

The data that support the findings of this study are available from the corresponding author upon reasonable request.

\section{ACKNOWLEDGEMENTS}

The financial support from the Swedish Energy Authority (project no. 32519-3), Volvo Cars AB (Göteborg, Sweden) and Scania CV AB (Södertälje, Sweden) is gratefully acknowledged.

\section{AUTHOR CONTRIBUTIONS}

X.F.W., K.J.K, S.B., M.B., U.W.G. and M.S.H. initiated the project. U.W.G. and M.S.H. supervised the study. K.J.K, X.F.W. and L.D.V aged the samples. X.F.W. and L.D.V performed the measurements and data analysis. P.L. performed the fuel uptake kinetics fitting. X.F.W. wrote the manuscript with the help of the other authors.

\section{ADDITIONAL INFORMATION}

Competing interests: The authors declare no competing interests.

Publisher's note: Springer Nature remains neutral with regard to jurisdictional claims in published maps and institutional affiliations.

\section{REFERENCES}

1. Akhlaghi, S., Gedde, U. W., Hedenqvist, M. S., Braña, M. T. C. \& Bellander, M. Deterioration of automotive rubbers in liquid biofuels: a review. Renew. Sust. Energ. Rev. 43, 1238-1248 (2015).

2. Akhlaghi, S., Hedenqvist, M. S., Braña, M. C., Bellander, M. \& Gedde, U. W. Deterioration of acrylonitrile butadiene rubber in rapeseed biodiesel. Polym. Degrad. Stab. 111, 211-222 (2015).

3. Knothe, G., Krahl, J. \& Van Gerpen, J. The Biodiesel Handbook. Academic Press and AOCS Press (2010).

4. Böhning, M., Niebergall, U., Adam, A. \& Stark, W. Impact of biodiesel sorption on mechanical properties of polyethylene. Polym. Test. 34, 17-24 (2014).

5. Böhning, M., Niebergall, U., Zanotto, M. \& Wachtendorf, V. Impact of biodiesel sorption on tensile properties of PE-HD for container applications. Polym. Test. 50, 315-324 (2016).

6. Van Rheinberg, O., Hoffmann, H., Jaschinski, C. \& Koch, W. Stability of fuels containing current and future biofuels. MTZ Worldw. 73, 12-18 (2012).
7. Chandran, D. et al. Compatibility of biodiesel fuel with metals and elastomers in fuel delivery system of a diesel engine. J. OIL PALM Res. 28, 64-73 (2016).

8. Xylia, M. \& Silveira, S. On the road to fossil-free public transport: the case of Swedish bus fleets. Energy Policy 100, 397-412 (2017).

9. Richaud, E., Djouani, F., Fayolle, B., Verdu, J. \& Flaconneche, B. New insights in polymer-biofuels interaction. Oil \& Gas. Sci. Technol. d'IFP Energ. Nouv. 70, 317-333 (2015).

10. Akhlaghi, S. et al. Degradation of carbon-black-filled acrylonitrile butadiene rubber in alternative fuels: Transesterified and hydrotreated vegetable oils. Polym. Degrad. Stab. 123, 69-79 (2016).

11. Østerstrøm, F. F. et al. Oxidation stability of rapeseed biodiesel/petroleum diesel blends. Energy Fuels 30, 344-351 (2016).

12. Akhlaghi, S. et al. Degradation of fluoroelastomers in rapeseed biodiesel at different oxygen concentrations. Polym. Degrad. Stab. 136, 10-19 (2017).

13. Richaud, E., Fayolle, B., Verdu, J. \& Rychlý, J. Co-oxidation kinetic model for the thermal oxidation of polyethylene-unsaturated substrate systems. Polym. Degrad. Stab. 98, 1081-1088 (2013).

14. Fazal, M., Haseeb, A. \& Masjuki, H. Biodiesel feasibility study: an evaluation of material compatibility; performance; emission and engine durability. Renew. Sust. Energ. Rev. 15, 1314-1324 (2011).

15. Mofijur, M. et al. Effect of biodiesel from various feedstocks on combustion characteristics, engine durability and materials compatibility: a review. Renew. Sust. Energ. Rev. 28, 441-455 (2013).

16. Sorate, K. A. \& Bhale, P. V. Biodiesel properties and automotive system compatibility issues. Renew. Sust. Energ. Rev. 41, 777-798 (2015).

17. Page, I. Polyamides As Engineering Thermoplastic Materials. Vol. 11 (iSmithers Rapra Publishing, 2000).

18. Kallio, K. J. \& Hedenqvist, M. S. Ageing properties of polyamide-12 pipes exposed to fuels with and without ethanol. Polym. Degrad. Stab. 93, 1846-1854 (2008).

19. Kallio, K. J., Nageye, A. S. \& Hedenqvist, M. S. Ageing properties of car fuel-lines; accelerated testing in 'close-to-real' service conditions. Polym. Test. 29, 41-48 (2010).

20. Wei, X.-F. et al. Long-term performance of a polyamide-12-based fuel line with a thin poly(ethylene-co-tetrafluoroethylene) (ETFE) inner layer exposed to bio- and petroleum diesel. Polym. Degrad. Stab. 156, 170-179 (2018).

21. Choudhury, P. S. \& Mallick, P. Effect of Biodiesel on The Tensile Properties of Nylon-6. Report No. 0148-7191 (SAE Technical Paper, 2012).

22. Nascimento, R. A. \& de Souza, A. M. C. Mechanical properties of polyamide 12 after exposed to biodiesel. AIP Conference Proceedings 1779, 070010 (2016).

23. Wei, X.-F. et al. Long-term performance of polyamide-based multilayer (bio) diesel fuel lines aged under 'in-vehicle' conditions. Polym. Degrad. Stab. 144, 100-109 (2017).

24. Stoffers, N., Brandl, F., Linssen, J. \& Franz, R. Development and validation of analytical methods for monomeric and oligomeric migrants from nylon 12 packaging materials. Food Addit. Contam. 20, 410-416 (2003).

25. Chernev, B. S. \& Eder, G. C. Side effects in the application of polyamide 6 barrier materials for fuel tanks. J. Appl. Polym. Sci. 127, 230-236 (2013).

26. Shan, G.-F. et al. Multiple melting behaviour of annealed crystalline polymers. Polym. Test. 29, 273-280 (2010).

27. Bonnet, M., Rogausch, K.-D. \& Petermann, J. The endothermic 'annealing peak' of poly (phenylene sulphide) and poly (ethylene terephthalate). Colloid Polym. Sci. 277, 513-518 (1999).

28. Liu, T. et al. Morphology, thermal and mechanical behavior of polyamide 6/ layered-silicate nanocomposites. Compos. Sci. Technol. 63, 331-337 (2003).

29. Celina, M. C. Review of polymer oxidation and its relationship with materials performance and lifetime prediction. Polym. Degrad. Stab. 98, 2419-2429 (2013).

30. Dong, W. \& Gijsman, P. Influence of temperature on the thermo-oxidative degradation of polyamide 6 films. Polym. Degrad. Stab. 95, 1054-1062 (2010).

31. Wei, X.-F. et al. Diffusion-limited oxidation of polyamide: Three stages of fracture behavior. Polym. Degrad. Stab. 154, 73-83 (2018).

32. Okamba-Diogo, O. et al. Investigation of polyamide 11 embrittlement during oxidative degradation. Polymers. 82, 49-56 (2016).

33. Gogolewski, S., Czerntawska, K. \& Gastorek, M. Effect of annealing on thermal properties and crystalline structure of polyamides. Nylon 12 (polylaurolactam). Colloid Polym. Sci. 258, 1130-1136 (1980).

34. Zhang, J. \& Adams, A. Understanding thermal aging of non-stabilized and stabilized polyamide 12 using $1 \mathrm{H}$ solid-state NMR. Polym. Degrad. Stab. 134, 169-178 (2016).

35. Gijsman, P., Dong, W., Quintana, A. \& Celina, M. Influence of temperature and stabilization on oxygen diffusion limited oxidation profiles of polyamide 6. Polym. Degrad. Stab. 130, 83-96 (2016).

36. Frisch, H. Sorption and transport in glassy polymers-a review. Polym. Eng. Sci. 20, 2-13 (1980). 
37. Hedenqvist, M. \& Gedde, U. Parameters affecting the determination of transport kinetics data in highly swelling polymers above Tg. Polymers. 40, 2381-2393 (1999).

38. Hedenqvist, M. \& Gedde, U. W. Diffusion of small-molecule penetrants in semicrystalline polymers. Prog. Polym. Sci. 21, 299-333 (1996).

Open Access This article is licensed under a Creative Commons Attribution 4.0 International License, which permits use, sharing, adaptation, distribution and reproduction in any medium or format, as long as you give appropriate credit to the original author(s) and the source, provide a link to the Creative
Commons license, and indicate if changes were made. The images or other third party material in this article are included in the article's Creative Commons license, unless indicated otherwise in a credit line to the material. If material is not included in the article's Creative Commons license and your intended use is not permitted by statutory regulation or exceeds the permitted use, you will need to obtain permission directly from the copyright holder. To view a copy of this license, visit http://creativecommons. org/licenses/by/4.0/.

c The Author(s) 2019 